\title{
Existence results for abstract semilinear evolution differential inclusions with nonlocal conditions
}

Tao Zhu ${ }^{*}$, Chao Song ${ }^{1}$ and Gang $\mathrm{Li}^{2}$

\section{"Correspondence:}

zhutaoyzu@yahoo.com.cn

${ }^{1}$ Department of Mathematics and

Physics, Nanjing Institute of

Technology, Nanjing, 211100, P.R.

China

Full list of author information is

available at the end of the article

\begin{abstract}
In this paper, we use a new method to study semilinear evolution differential inclusions with nonlocal conditions in Banach spaces. We derive conditions for $F$ and $g$ for the existence of mild solutions. The results obtained here improve and generalize many known results.
\end{abstract}

MSC: $34 \mathrm{~A} 60 ; 34 \mathrm{G} 20$

Keywords: semilinear evolution differential inclusions; mild solutions; measure of noncompactness; upper semicontinuous

\section{Introduction}

In this paper, we discuss the nonlocal initial value problem

$$
\left\{\begin{array}{l}
x^{\prime}(t) \in A x(t)+F(t, x(t)), \quad t \in I=[0,1], \\
x(0)=g(x),
\end{array}\right.
$$

where $A$ is the infinitesimal generator of a strongly continuous semigroup of bounded linear operators (i.e., $C_{0}$-semigroup) $T(t)$ in a Banach space $X$, and $F:[0,1] \times X \rightarrow P_{c}(X)$, $g: C([0,1] ; X) \rightarrow X$ are given $X$-valued functions.

The study of nonlocal evolution equations was initiated by Byszewski [1]. Since these represent mathematical models of various phenomena in physics, Byszewski's work was followed by many others [2-7]. Subsequently, many authors have contributed to the study of the differential inclusions (1.1). Differential inclusions (1.1) were first considered by Aizicovici and Gao [8] when $g$ and $T(t)$ are compact. In [9-12] the semilinear evolution differential inclusions (1.1) were discussed when $A$ generates a compact semigroup. Xue and Song [13] established the existence of mild solutions to the differential inclusions (1.1) when $A$ generates an equicontinuous semigroup and $F(t, \cdot)$ is l.s.c. for a.e. $t \in[0,1]$. In [14] the author proved the existence of mild solutions of the differential inclusions (1.1) when $A$ generates an equicontinuous semigroup and a Banach space $X$ which is separable and uniformly smooth. In [15] Zhu and Li studied the differential inclusions (1.1) when $F$ admits a strongly measurable selector. In [16] the differential inclusions (1.1) were discussed when $\{A(t)\}$ is a family of linear (not necessarily bounded) operators. In [17] local and global existence results for differential inclusions with infinite delay in a Banach space were considered. Benchohra and Ntouyas [18] studied the second-order initial value problems for

(c) 2013 Zhu et al.; licensee Springer. This is an Open Access article distributed under the terms of the Creative Commons Attribution License (http://creativecommons.org/licenses/by/2.0), which permits unrestricted use, distribution, and reproduction in any medium, provided the original work is properly cited. 
delay integrodifferential inclusions. In $[19,20]$ the impulsive multivalued semilinear neutral functional differential inclusions were discussed in the case that the linear semigroup $T(t)$ is compact. The purpose of this paper is to continue the study of these authors. By using a new method, we prove the existence results of mild solutions for (1.1) under the following conditions of $g$ and $T(t): g$ is either compact or Lipschitz continuous and $T(t)$ is an equicontinuous semigroup. So, our work extends and improves many main results such as those in $[8-12,14,15]$.

The organization of this work is as follows. In Section 2, we recall some definitions and facts about set-valued analysis and the measure of noncompactness. In Section 3, we give the existence of mild solutions of the nonlocal initial value problem (1.1). In Section 4, an example is given to show the applications of our results.

\section{Preliminaries}

Let $(X,\|\cdot\|)$ be a real Banach space. Let $P_{c}(X)=\{A \subseteq X$ : nonempty, closed, convex $\}$. A multivalued map $G: X \rightarrow X$ is convex (closed) valued if $G(x)$ is convex (closed) for all $x \in X$. We say that $G$ is bounded on bounded sets if $G(B)$ is bounded in $X$ for each bounded set $B$ of $X$. The map $G$ is called upper semicontinuous (u.s.c.) on $X$ if for each $x_{0} \in X$ the set $G\left(x_{0}\right)$ is a nonempty, closed subset of $X$, and if for each open set $N$ of $X$ containing $G\left(x_{0}\right)$, there exists an open neighborhood $M$ of $x_{0}$ such that $G(M) \subseteq N$. Also, $G$ is said to be completely continuous if $G(B)$ is relatively compact for every bounded subset $B \subseteq X$. If the multivalued map $G$ is completely continuous with nonempty compact values, then $G$ is u.s.c. if and only if $G$ has a closed graph (i.e., $x_{n} \rightarrow x_{0}, y_{n} \rightarrow y_{0}, y_{n} \in G\left(x_{n}\right)$ imply $\left.y_{0} \in G\left(x_{0}\right)\right)$. Moreover, the following conclusions hold. Let $D \subset X$ and $G(x)$ be closed for all $x \in D$, if $G$ is u.s.c. and $D$ is closed, then $\operatorname{graph}(G)$ is closed. If $\overline{G(D)}$ is compact and $D$ is closed, then $G$ is u.s.c. if and only if $\operatorname{graph}(G)$ is closed. Finally, we say that $G$ has a fixed point if there exists $x \in X$ such that $x \in G(x)$.

We denote by $C([0,1] ; X)$ the space of $X$-valued continuous functions on $[0,1]$ with the norm $\|x\|=\sup \{\|x(t)\| ; t \in[0,1]\}$, and by $L^{1}(0,1 ; X)$ the space of $X$-valued Bochner functions on $[0,1]$ with the norm $\|x\|=\int_{0}^{1}\|x(s)\| d s$.

A $C_{0}$-semigroup $T(t)$ is said to be compact if $T(t)$ is compact for any $t>0$. If the semigroup $T(t)$ is compact, then $t \rightarrow T(t) x$ is equicontinuous at all $t>0$ with respect to $x$ in all bounded subsets of $X$; i.e., the semigroup $T(t)$ is equicontinuous. If $A$ is the generator of an analytic semigroup $T(t)$ or a differentiable semigroup $T(t)$, then $T(t)$ is an equicontinuous $C_{0}$-semigroup (see [21]). In this paper, we suppose that $A$ generates an equicontinuous semigroup $T(t)$ on $X$. Since no confusion may occur, we denote by $\alpha$ the Hausdorff measure of noncompactness on both $X$ and $C([0,1] ; X)$.

Definition 2.1 A function $x \in C([0,1] ; X)$ is a mild solution of (1.1) if

(1) $x(t)=T(t) g(x)+\int_{0}^{t} T(t-s) v(s) d s$,

(2) $x(0)=g(x)$, where $v \in S_{F, x}=\left\{v \in L^{1}(I, X): v(t) \in F(t, x(t))\right\}$.

To prove the existence results in this paper, we need the following lemmas.

Lemma 2.2 [22] If $W \subseteq C([0,1] ; X)$ is bounded, then $\alpha(W(t)) \leq \alpha(W)$ for all $t \in[0,1]$, where $W(t)=\{x(t) ; x \in W\} \subseteq X$. Furthermore, if $W$ is equicontinuous on $[0,1]$, then $\alpha(W(t))$ is continuous on $[0,1]$, and $\alpha(W)=\sup \{\alpha(W(t)) ; t \in[0,1]\}$. 
Lemma 2.3 [22] If $\left\{W_{n}\right\}_{n=1}^{+\infty}$ is a decreasing sequence of bounded closed nonempty subsets of $X$ and $\lim _{n \rightarrow+\infty} \alpha\left(W_{n}\right)=0$, then $\bigcap_{n=1}^{+\infty} W_{n}$ is nonempty and compact in $X$.

Lemma 2.4 [23] If $\left\{u_{n}\right\}_{n=1}^{\infty} \subset L^{1}(0,1 ; X)$ is uniformly integrable, then $\alpha\left(\left\{u_{n}(t)\right\}_{n=1}^{\infty}\right)$ is measurable and

$$
\alpha\left(\left\{\int_{0}^{t} u_{n}(s) d s\right\}_{n=1}^{\infty}\right) \leq 2 \int_{0}^{t} \alpha\left(\left\{u_{n}(s)\right\}_{n=1}^{\infty}\right) d s .
$$

Lemma 2.5 [24] If the semigroup $T(t)$ is equicontinuous and $\eta \in L^{1}\left(0,1 ; \Re^{+}\right)$, then the set $\left\{t \rightarrow \int_{0}^{t} T(t-s) x(s) d s ; x \in L^{1}\left(0,1 ; \Re^{+}\right),\|x(s)\| \leq \eta(s)\right.$, for a.e. $\left.s \in[0,1]\right\}$ is equicontinuous on $[0,1]$.

Lemma 2.6 [25] If $W$ is bounded, then for each $\varepsilon>0$, there is a sequence $\left\{u_{n}\right\}_{n=1}^{\infty} \subseteq W$ such that

$$
\alpha(W) \leq 2 \alpha\left(\left\{u_{n}\right\}_{n=1}^{\infty}\right)+\varepsilon .
$$

A countable set $\left\{f_{n}\right\}_{n=1}^{\infty} \subset L^{1}(0,1 ; X)$ is said to be semicompact if

(a) it is integrably bounded: $\left\|f_{n}(t)\right\| \leq \omega(t)$ for a.e. $t \in[0,1]$ and every $n \geq 1$, where

$$
\omega(\cdot) \in L^{1}\left(0,1 ; \Re^{+}\right)
$$

(b) the set $\left\{f_{n}(t)\right\}_{n=1}^{\infty}$ is relatively compact for a.e. $t \in[0,1]$.

Lemma 2.7 [26] Every semicompact set is weakly compact in the space $L^{1}(0,1 ; X)$.

Lemma $2.8[16,26]$ If $\left\{f_{n}\right\}_{n=1}^{\infty} \subset L^{1}(0,1 ; X)$ is semicompact, then $\left\{\int_{0}^{t} T(t-s) f_{n}(s) d s\right\}_{n=1}^{\infty}$ is relatively compact in $C([0,1] ; X)$ and, moreover, if $f_{n} \rightarrow f_{0}$, then

$$
\int_{0}^{t} T(t-s) f_{n}(s) d s \rightarrow \int_{0}^{t} T(t-s) f_{0}(s) d s
$$

as $n \rightarrow \infty$.

The map $F: W \subseteq X \rightarrow X$ is said to be $\alpha$ contraction if there exists a positive constant $k<1$ such that

$$
\alpha(F(Q)) \leq k \alpha(Q)
$$

for any bounded closed subset $Q \subseteq W$.

Lemma 2.9 [27-30] (Fixed point theorem) If $W \subseteq X$ is a nonempty, bounded, closed, convex and compact subset, the map $F: W \rightarrow 2^{W}$ is upper semicontinuous with $F(x) a$ nonempty, closed, convex subset of $W$ for each $x \in W$, then $F$ has at least one fixed point in $W$.

Lemma 2.10 [26] (Fixed point theorem) If $W \subseteq X$ is nonempty, bounded, closed and convex, the map $F: W \rightarrow 2^{W}$ is a closed $\alpha$ contraction map with $F(x)$ a nonempty, convex and compact subset of $W$ for each $x \in W$, then $F$ has at least one fixed point in $W$. 


\section{Main results}

In this section, by using the measure of noncompactness and fixed point theorems, we give the existence results of the nonlocal initial value problem (1.1). Here we list the following hypotheses.

(1) The $C_{0}$ semigroup $T(t)$ generated by $A$ is equicontinuous. We denote $N=\sup \{\|T(t)\| ; t \in[0,1]\}$.

(2) $g: C([0,1] ; X) \rightarrow X$ is continuous and compact, there exist positive constants $c$ and $d$ such that $\|g(x)\| \leq c\|x\|+d, \forall x \in C([0,1] ; X)$.

(3) The multivalued operator $F:[0,1] \times X \rightarrow P_{c}(X)$ satisfies the hypotheses:

$$
\begin{aligned}
& t \rightarrow F(t, x) \text { is measurable for every } x \in X ; \\
& x \rightarrow F(t, x) \text { is u.s.c. for a.e. } t \in[0,1] ;
\end{aligned}
$$

the set $S_{F, x}=\left\{v \in L^{1}(I, X): v(t) \in F(t, x(t))\right.$; for a.e. $\left.t \in[0,1]\right\}$ is nonempty.

(4) There exists $L \in L^{1}\left(0,1 ; \Re^{+}\right)$such that for any bounded $D \subset X$,

$$
\alpha(F(t, D)) \leq L(t) \alpha(D)
$$

for a.e. $t \in[0,1]$.

(5) There exist a function $m \in L^{1}\left(0,1 ; \Re^{+}\right)$and a nondecreasing continuous function $\Omega: \Re^{+} \rightarrow \Re^{+}$such that

$$
\|F(t, x)\| \leq m(t) \Omega(\|x\|)
$$

for all $x \in X$, and a.e. $t \in[0,1]$.

Remark 3.1 If $\operatorname{dim} X<\infty$, then $S_{F, x} \neq \emptyset$ for each $x \in C([0,1] ; X)$ (see Lasota and Opial [31]). If $\operatorname{dim} X=\infty$ and $x \in C([0,1] ; X)$, then the set $S_{F, x}$ is nonempty if and only if the function $Y:[0,1] \rightarrow \Re$ defined by $Y(t)=\inf \{\|v\|: v \in F(t, x(t))\}$ belongs to $L^{1}\left(0,1 ; \Re^{+}\right)$(see $\mathrm{Hu}$ and Papageorgiou [32]).

The following lemma plays a crucial role in the proof of the main theorem.

Lemma 3.2 [26] Under assumptions (3)-(5), if we consider sequences $\left\{x_{n}\right\}_{n=1}^{\infty} \subset C([0,1] ; X)$ and $\left\{v_{n}\right\}_{n=1}^{\infty} \subset L^{1}(0,1 ; X)$, where $v_{n} \in S_{F, x_{n}}$, such that $x_{n} \rightarrow x, v_{n} \rightarrow v$, then $v \in S_{F, x}$.

Now we give the existence results under the above hypotheses.

Theorem 3.3 If (1)-(5) are satisfied, then there is at least one mild solution for (1.1) provided that there exists a constant $R$ with

$$
\int_{0}^{1} m(s) d s<\int_{N(c R+d)}^{R} \frac{1}{N \Omega(s)} d s .
$$

Proof Define the operator $\Gamma: C([0,1] ; X) \rightarrow C([0,1] ; X)$ by

$$
(\Gamma x)(t)=\left\{y(t) \in C([0,1] ; X): y(t)=T(t) g(x)+\int_{0}^{t} T(t-s) v(s) d s ; v \in S_{F, x}\right\} .
$$

We shall show that the multivalued $\Gamma$ has at least one fixed point. The fixed point is then a mild solution of the problem (1.1). 
(1) We contract a bounded, convex, closed and compact set $W \subset C([0,1] ; X)$ such that $\Gamma$ maps $W$ into itself.

In view of (3.1), we know there exists a constant $\eta>0$ such that

$$
\int_{0}^{1} m(s) d s<\int_{T_{0}+\eta}^{R} \frac{1}{N \Omega(s)} d s
$$

where $T_{0}=N(c R+d)$.

Then there exists a positive integer $K$ such that

$$
\int_{T_{0}+\eta}^{T_{0}+K \eta} \frac{1}{N \Omega(s)} d s<\int_{0}^{1} m(s) d s \leq \int_{T_{0}+\eta}^{T_{0}+(K+1) \eta} \frac{1}{N \Omega(s)} d s .
$$

Hence, we get $0=t_{0}<t_{1}<t_{2}<\cdots<t_{K-1}<t_{K}=1$ such that

$$
\begin{aligned}
& \int_{0}^{t_{1}} m(s) d s=\int_{T_{0}+\eta}^{T_{0}+2 \eta} \frac{1}{N \Omega(s)} d s, \\
& \int_{t_{1}}^{t_{2}} m(s) d s=\int_{T_{0}+2 \eta}^{T_{0}+3 \eta} \frac{1}{N \Omega(s)} d s, \\
& \ldots, \\
& \int_{t_{K-2}}^{t_{K-1}} m(s) d s=\int_{T_{0}+(K-1) \eta}^{T_{0}+K \eta} \frac{1}{N \Omega(s)} d s, \\
& \int_{t_{K-1}}^{1} m(s) d s \leq \int_{T_{0}+K \eta}^{T_{0}+(K+1) \eta} \frac{1}{N \Omega(s)} d s .
\end{aligned}
$$

We denote $W_{0}=\left\{x \in C([0,1] ; X),\|x(t)\|_{i}=\sup \left\{\|x(t)\|: t \in\left[t_{i-1}, t_{i}\right]\right\} \leq T_{0}+i \eta, i=\right.$ $1,2, \ldots, K\}$, then $W_{0} \subseteq C([0,1] ; X)$ is nonempty, bounded, closed and convex.

For any $x \in W_{0}$, we have

$$
(\Gamma x)(t)=\left\{y(t): y(t)=T(t) g(x)+\int_{0}^{t} T(t-s) v(s) d s ; v(t) \in S_{F, x}\right\} .
$$

Therefore

$$
\begin{aligned}
\|y(t)\| & \leq\|T(t) g(x)\|+\left\|\int_{0}^{t} T(t-s) v(s) d s\right\| \leq N(c\|x\|+d)+N \int_{0}^{t} m(s) \Omega(\|x(s)\|) d s \\
& \leq N\left(c\left(T_{0}+K \eta\right)+d\right)+N \int_{0}^{t} m(s) \Omega(\|x(s)\|) d s \\
& \leq N(c R+d)+N \int_{0}^{t} m(s) \Omega(\|x(s)\|) d s \leq T_{0}+N \int_{0}^{t} m(s) \Omega(\|x(s)\|) d s,
\end{aligned}
$$

and

$$
\begin{aligned}
\|y\|_{i} & =\sup \left\{\|y(t)\|: t \in\left[t_{i-1}, t_{i}\right]\right\} \\
& \leq \sup \left\{T_{0}+N \int_{0}^{t} m(s) \Omega(\|x(s)\|) d s: t \in\left[t_{i-1}, t_{i}\right]\right\}
\end{aligned}
$$




$$
\begin{aligned}
\leq & T_{0}+N \int_{0}^{t_{i}} m(s) \Omega(\|x(s)\|) d s \\
\leq & T_{0}+N\left[\int_{0}^{t_{1}} m(s) \Omega(\|x(s)\|) d s+\int_{t_{1}}^{t_{2}} m(s) \Omega(\|x(s)\|) d s\right. \\
& \left.+\cdots+\int_{t_{i-1}}^{t_{i}} m(s) \Omega(\|x(s)\|) d s\right] \\
\leq & T_{0}+N\left[\int_{0}^{t_{1}} m(s) d s \Omega\left(T_{0}+\eta\right)+\int_{t_{1}}^{t_{2}} m(s) d s \Omega\left(T_{0}+2 \eta\right)\right. \\
& \left.+\cdots+\int_{t_{i-1}}^{t_{i}} m(s) d s \Omega\left(T_{0}+i \eta\right)\right] \\
\leq & T_{0}+N\left[\int_{T_{0}+\eta}^{T_{0}+2 \eta} \frac{1}{N \Omega(s)} d s \Omega\left(T_{0}+\eta\right)+\int_{T_{0}+2 \eta}^{T_{0}+3 \eta} \frac{1}{N \Omega(s)} d s \Omega\left(T_{0}+2 \eta\right)\right. \\
& \left.+\cdots+\int_{T_{0}+i \eta}^{T_{0}+(i+1) \eta} \frac{1}{N \Omega(s)} d s \Omega\left(T_{0}+i \eta\right)\right] \\
\leq & T_{0}+i \eta
\end{aligned}
$$

which implies $\Gamma: W_{0} \rightarrow 2^{W_{0}}$ is a bounded operator.

Define $W_{1}=\overline{\operatorname{conv}} \Gamma\left(W_{0}\right)$, where $\overline{\operatorname{conv}}$ means the closure of the convex hull in $C([0,1] ; X)$. Then $W_{1} \subset C([0,1] ; X)$ is nonempty bounded closed convex on $[0,1]$ with $W_{1} \subseteq W_{0}$. Let $W_{n+1}=\overline{\operatorname{conv}} \Gamma\left(W_{n}\right)$ for all $n \geq 1$. Similarly to the above discussions, we know that $W_{n+1} \subseteq W_{n}$ for $n=1,2, \ldots$ as $W_{1} \subseteq W_{0}$ and $W_{1}, W_{2}, \ldots$ are both nonempty, closed, bounded and convex. Thus $\left\{W_{n}\right\}_{n=1}^{+\infty}$ is a decreasing sequence consisting of subsets of $C([0,1] ; X)$. Moreover, set

$$
W=\bigcap_{n=1}^{+\infty} W_{n},
$$

then $W$ is a convex, closed and bounded subset of $C([0,1] ; X)$ and $\Gamma(W) \subseteq W$.

Now, we claim that $W$ is nonempty and compact in $C([0,1] ; X)$. To do so, from Lemma 2.6, we know for arbitrary given $\epsilon>0$, there exist sequences $\left\{v_{n}\right\}_{n=1}^{+\infty} \subset S_{F, W_{n}}$ such that

$$
\begin{aligned}
\alpha\left(W_{n+1}(t)\right) & =\alpha\left(\left(\Gamma W_{n}\right)(t)\right) \\
& \leq 2 \alpha\left(\int_{0}^{t} T(t-s) v_{n}(s)_{n=1}^{\infty} d s\right)+\varepsilon \\
& \leq 4 \int_{0}^{t} \alpha\left(T(t-s) v_{n}(s)_{n=1}^{\infty}\right) d s+\varepsilon \\
& \leq 4 N \int_{0}^{t} \alpha\left(v_{n}(s)_{n=1}^{\infty}\right) d s+\varepsilon \\
& \leq 4 N \int_{0}^{t} \alpha\left(F\left(s, W_{n}(s)\right) d s+\varepsilon\right. \\
& \leq 4 N \int_{0}^{t} L(s) \alpha\left(W_{n}(s)\right) d s+\varepsilon .
\end{aligned}
$$


Since this is true for arbitrary $\epsilon>0$, we have

$$
\alpha\left(W_{n+1}(t)\right) \leq 4 N \int_{0}^{t} L(s) \alpha\left(W_{n}(s)\right) d s
$$

Because $W_{n}$ is decreasing for $n$, we can define

$$
\mu(t)=\lim _{n \rightarrow+\infty} \alpha\left(\left(W_{n}\right)(t)\right)
$$

Let $n \rightarrow+\infty$, we have

$$
\mu(t) \leq 4 N \int_{0}^{t} L(s) \mu(s) d s
$$

It implies that $\mu(t)=0$ for all $t \in[0,1]$. By Lemma 2.2, we know that $\lim _{n \rightarrow+\infty} \alpha\left(W_{n}\right)=0$. Using Lemma 2.3, we obtain $W=\bigcap_{n=1}^{+\infty} W_{n}$ is nonempty and compact in $C([0,1] ; X)$.

(2) We shall show that $\Gamma$ is closed on $W$ with closed convex values. It is very easy to see that $\Gamma$ has convex values.

Let us now verity that graph $(\Gamma)$ is closed. Let $\left\{x_{n}\right\}_{n=1}^{\infty} \subset W$ with $x_{n} \rightarrow x$ in $C([0,1] ; X)$, and $y_{n} \in \Gamma x_{n}$ with $y_{n} \rightarrow y$ in $C([0,1] ; X)$. Moreover, let $\left\{v_{n}\right\}_{n=1}^{\infty} \subset L^{1}(0,1 ; X)$ be a sequence such that $v_{n} \in S_{F, x_{n}}$ for any $n \geq 1$, and

$$
y_{n}(t)=T(t) g\left(x_{n}\right)+\int_{0}^{t} T(t-s) v_{n}(s) d s
$$

As $x_{n} \rightarrow x$ in $C([0,1] ; X)$, we know that $\left\{x_{n}\right\}_{n=1}^{\infty}$ is a bounded set of $C([0,1] ; X)$, we denote $R_{x}=\sup \left\{\left\|x_{n}\right\|: n=1,2, \ldots\right\}$.

From hypothesis (5), we obtain

$$
\left\|v_{n}(t)\right\| \leq \| F\left(t, x_{n}(t) \| \leq m(t) \Omega\left(\left\|x_{n}\right\|\right) \leq m(t) \Omega\left(R_{x}\right) .\right.
$$

Then we have the set $\left\{v_{n}\right\}_{n=1}^{\infty}$ is integrably bounded for a.e. $t \in[0,1]$.

From hypothesis (4), we know

$$
\alpha\left(\left\{v_{n}(t)\right\}_{n=1}^{\infty}\right) \leq \alpha\left(F\left(t,\left\{x_{n}(t)\right\}_{n=1}^{\infty}\right)\right) \leq L(t) \alpha\left(\left\{x_{n}(t)\right\}_{n=1}^{\infty}\right)=0
$$

for a.e. $t \in[0,1]$. Then the set $\left\{v_{n}(t)\right\}_{n=1}^{\infty}$ is relatively compact for a.e. $t \in[0,1]$.

So, the set $\left\{v_{n}(t)\right\}_{n=1}^{\infty}$ is semicompact. By applying Lemma 2.7, it yields that $\left\{v_{n}(t)\right\}_{n=1}^{\infty}$ is weakly compact in $L^{1}(0,1 ; X)$. We get that there exists $v \in L^{1}(0,1 ; X)$ such that $v_{n} \rightarrow v$. Therefore, we infer that

$$
\int_{0}^{t} T(t-s) v_{n}(s) d s \rightarrow \int_{0}^{t} T(t-s) v(s) d s
$$

Further, we have

$$
y_{n}(t) \rightarrow T(t) g(x)+\int_{0}^{t} T(t-s) v(s) d s
$$


and hence

$$
y(t)=T(t) g(x)+\int_{0}^{t} T(t-s) v(s) d s .
$$

By Lemma 3.2, it implies that $v \in S_{F, x}$, i.e., $y \in \Gamma(x)$. Therefore $\operatorname{graph}(\Gamma)$ is closed. And hence $\Gamma$ has closed values on $W$.

(4) $\Gamma$ is u.s.c. on $W$.

Since $\overline{\Gamma W} \subseteq W$ is compact, $W$ is closed and $\operatorname{graph}(\Gamma)$ is closed, we can come to the conclusion that $\Gamma$ is u.s.c. (see [30]).

Finally, due to fixed point Lemma 2.9, $\Gamma$ has at least one point $x \in \Gamma(x)$, and $x$ is a mild solution to the semilinear evolution differential inclusions with the nonlocal conditions (1.1). Thus the proof is complete.

Remark 3.4 In [8-12] the authors discuss the nonlocal initial value problem (1.1) when $T(t)$ is compact. In [14] the existence of mild solutions of the differential inclusions (1.1) is proved when A generates an equicontinuous semigroup and Banach space $X$ is separable and uniformly smooth. In this paper, by using a new method, we prove the operator $\Gamma$ maps compact set $W$ into itself. We do not impose any restriction on the coefficient $L(t)$, and we only require $T(t)$ to be an equicontinuous semigroup. So, Theorem 3.3 generalizes and improves the related results in $[8-12,14]$.

Theorem 3.5 [15] If (1)-(5) are satisfied, then there is at least one mild solution for (1.1) provided that there exists a constant $R>0$ such that

$$
N(c R+d)+N \int_{0}^{1} m(s) d s \Omega(R) \leq R
$$

Proof In view of (3.4), we get

$$
\int_{0}^{1} m(s) d s \leq \frac{R-N(c R+d)}{N \Omega(R)}<\int_{N(c R+d)}^{R} \frac{1}{N \Omega(s)} d s .
$$

From Theorem 3.3, the nonlocal initial value problem (1.1) has at least one mild solution.

Remark 3.6 If $N=1, c=\frac{1}{3}, d=0, \Omega(x)=x$ and $\int_{0}^{1} m(s) d s=1$. We cannot obtain a constant $R$ such that

$$
\frac{1}{3} R+R \leq R
$$

By using Theorem 3.5, we do not know whether or not equation (1.1) has a mild solution. But we know there exists a constant $R=1$ such that

$$
\int_{0}^{1} m(s) d s=1<\ln 3=\int_{N(c R+d)}^{R} \frac{1}{N \Omega(s)} d s .
$$

So, Theorem 3.3 is better than Theorem 3.5. 
Theorem 3.7 [12] If (1)-(5) are satisfied and $\|g(x)\| \leq d$, then there is at least one mild solution for (1.1) provided that

$$
\int_{0}^{1} m(s) d s<\int_{d}^{+\infty} \frac{1}{N \Omega(s)} d s
$$

Proof In view of (3.5), we get there exists a constant $R$ such that

$$
\int_{0}^{1} m(s) d s<\int_{0 R+d}^{R} \frac{1}{N \Omega(s)} d s
$$

By Theorem 3.3, we complete the proof of this theorem.

Next, we give the existence result for (1.1) when $g$ is Lipschitz continuous.

We suppose that:

(6) There exists a constant $c \in \mathfrak{R}^{+}$such that $\|g(u)-g(v)\| \leq c\|u-v\|$ for all $u, v \in$ $C([0,1] ; X)$. Therefore, $\|g(x)\| \leq c\|x\|+d$, where $d=\|g(0)\|$.

Theorem 3.8 If (1) and (3)-(6) are satisfied and

$$
N c+4 N \int_{0}^{1} L(s) d s<1
$$

then there is at least one mild solution for (1.1) provided that there exists a constant $R$ satisfying

$$
\int_{0}^{1} m(s) d s<\int_{T_{0}}^{+\infty} \frac{1}{N \Omega(s)} d s
$$

Proof With the same arguments as given in the first portion of the proof of Theorem 3.3, we know $\Gamma: W_{0} \rightarrow 2^{W_{0}}$ is a bounded map with convex values and is closed on $W_{0}$.

Now, we prove the values of $\Gamma$ are compact in $C([0,1] ; X)$.

Let $x \in C([0,1] ; X)$ and $y_{n} \in \Gamma(x)$. To prove that $\Gamma(x)$ is compact, we have to show that $y_{n}$ has a subsequence converging to a point $y \in \Gamma(x)$. We have $v_{n} \in S_{F, x}$ such that

$$
y_{n}(t)=T(t) g(x)+\int_{0}^{t} T(t-s) v_{n}(s) d s
$$

From hypothesis (5), we obtain

$$
\left\|v_{n}(t)\right\| \leq\|F(t, x(t))\| \leq m(t) \Omega(\|x\|) .
$$

Then we have the set $\left\{v_{n}\right\}_{n=1}^{\infty}$ is integrably bounded for a.e. $t \in[0,1]$.

From hypothesis (4), we know

$$
\alpha\left(\left\{v_{n}(t)\right\}_{n=1}^{\infty}\right) \leq \alpha(F(t, x(t))) \leq L(t) \alpha(x(t))=0
$$

for a.e. $t \in[0,1]$. Then the set $\left\{v_{n}(t)\right\}_{n=1}^{\infty}$ is relatively compact for a.e. $t \in[0,1]$. 
So, the set $\left\{v_{n}(t)\right\}_{n=1}^{\infty}$ is semicompact. By applying Lemma 2.7, it yields that $\left\{v_{n}(t)\right\}_{n=1}^{\infty}$ is weakly compact in $L^{1}(0,1 ; X)$. We get that there exists $v \in L^{1}(0,1 ; X)$ such that $v_{n} \rightarrow v$. Therefore, we infer that

$$
\int_{0}^{t} T(t-s) v_{n}(s) d s \rightarrow \int_{0}^{t} T(t-s) v(s) d s,
$$

and

$$
\lim _{n \rightarrow+\infty} y_{n}(t)=T(t) g(x)+\int_{0}^{t} T(t-s) v(s) d s=y(t) .
$$

By Lemma 3.2, it implies that $v \in S_{F, x}$, i.e., $y \in \Gamma(x)$. Therefore $\Gamma$ has compact values.

Next, we prove $\Gamma$ is an $\alpha$ contraction map. For any $B \subseteq W_{0}$, we have

$$
\begin{aligned}
\alpha((\Gamma B)(t)) & =\alpha\left(T(t) g(B)+\int_{0}^{t} T(t-s) S_{F, B} d s\right) \\
& \leq N c \alpha(B)+\alpha\left(\int_{0}^{t} T(t-s) S_{F, B} d s\right) .
\end{aligned}
$$

From Lemma 2.6, we know for arbitrary given $\epsilon>0$, there exist sequences $\left\{v_{n}\right\}_{n=1}^{+\infty} \subset S_{F, B}$ such that

$$
\begin{aligned}
\alpha\left(\int_{0}^{t} T(t-s) S_{F, B} d s\right) & =2 \alpha\left(\int_{0}^{t} T(t-s) v_{n}(s)_{n=1}^{\infty} d s\right)+\varepsilon \\
& \leq 4 \int_{0}^{t} \alpha\left(T(t-s) v_{n}(s)_{n=1}^{\infty}\right) d s+\varepsilon \\
& \leq 4 N \int_{0}^{t} \alpha\left(v_{n}(s)_{n=1}^{\infty}\right) d s+\varepsilon \\
& \leq 4 N \int_{0}^{t} \alpha(F(s, B(s))) d s+\varepsilon \\
& \leq 4 N \int_{0}^{t} L(s) \alpha(B(s)) d s+\varepsilon \\
& \leq 4 N \alpha(B) \int_{0}^{1} L(s) d s+\varepsilon .
\end{aligned}
$$

Since this is true for arbitrary $\epsilon>0$, we have

$$
\alpha\left(\int_{0}^{t} T(t-s) S_{F, B} d s\right) \leq 4 N \alpha(B) \int_{0}^{1} L(s) d s .
$$

Therefore, we obtain

$$
\alpha(\Gamma B) \leq\left(N c+4 N \int_{0}^{1} L(s) d s\right) \alpha(B)
$$

Noting $N c+4 N \int_{0}^{t} L(s) d s<1$, therefore $\Gamma$ is an $\alpha$ contraction map.

Finally, due to Lemma 2.10, $\Gamma$ has at least one fixed point. This completes the proof. 


\section{An example}

In this section, as an application of our main results, an example is presented. We consider the following partial differential equation:

$$
\left\{\begin{array}{l}
\frac{\partial u(\zeta, t)}{\partial t}+\Sigma_{|\alpha| \leq 2 m} a_{\alpha}(\zeta) D^{\alpha} u(\zeta, t) \in f(t, u(\zeta, t)), \quad(\zeta, t) \in \Omega \times[0,1] \\
u(\zeta, t)=0, \quad(\zeta, t) \in \partial \Omega \times[0,1], \\
u(\zeta, 0)=\int_{\Omega} \int_{0}^{1} k(t, \zeta, \eta, u(\eta, t)) d t d \eta, \quad \zeta \in \Omega
\end{array}\right.
$$

where $\Omega$ is a bounded domain in $\Re^{n}$ with a smooth boundary $\partial \Omega, a_{\alpha}(\zeta)$ is a smooth real function on $\bar{\Omega}$.

We suppose that

(a) The differential operator $\Sigma_{|\alpha| \leq 2 m} a_{\alpha}(\zeta) D^{\alpha}$ is strongly elliptic [21].

(b) The function $k:[0,1] \times \Omega \times \Omega \times \Re \rightarrow \Re$ satisfies the following conditions:

(b $\left.\mathrm{b}_{1}\right) k(t, \zeta, \eta, r)$ is a continuous function about $r$ for a.e. $(t, \zeta, \eta) \in[0,1] \times \Omega \times \Omega$.

(b $\left.\mathrm{b}_{2}\right) k(t, \zeta, \eta, r)$ is measurable about $(t, \zeta, \eta)$ for each fixed $r \in \Re$.

$\left(\mathrm{b}_{3}\right)$ For any $R>0$, there is $\beta_{R} \in L^{1}\left([0,1] \times \Omega \times \Omega \times \Re\right.$; $\left.\Re^{+}\right)$such that

$$
\left|k(t, \zeta, \eta, r)-k\left(t, \zeta^{\prime}, \eta, r\right)\right| \leq \beta_{R}\left(t, \zeta, \zeta^{\prime}, \eta\right)
$$

for all $(t, \zeta, \eta, r),\left(t, \zeta^{\prime}, \eta, r\right) \in([0,1] \times \Omega \times \Omega \times \Re)$ with $|r| \leq R$, and

$$
\lim _{\Delta \zeta \rightarrow 0} \int_{\Omega} \int_{0}^{1} \beta_{R}(t, \zeta, \Delta \zeta, \eta) d t d \eta=0
$$

uniformly for $\zeta \in \Omega$.

$\left(\mathrm{b}_{4}\right)$ There exist $a(\cdot) \in L(0,1)$ and $d(\cdot) \in L^{2}\left([0,1] \times \Omega \times \Omega, \Re^{+}\right)$such that

$$
|k(t, \zeta, \eta, r)| \leq a(t) r+d(t, \zeta, \eta)
$$

for all $(t, \zeta, \eta, r) \in([0,1] \times \Omega \times \Omega \times \Re)$.

Let $D(A)=H^{2 m} \cap H_{0}^{m}(\Omega)$ and $A u(\zeta)=-\Sigma_{|\alpha| \leq 2 m} a_{\alpha}(\zeta) D^{\alpha} u(\zeta, \cdot)$, then $A$ generates an analytic semigroup on $X=L^{2}(\Omega)$ ([21]). We suppose

$$
g(u)(\zeta)=\int_{\Omega} \int_{0}^{1} k(t, \zeta, \eta, u(\eta, t)) d t d \eta
$$

From [33], we obtain $g$ satisfies hypothesis (2).

Then equation (4.1) can be regarded as the following nonlocal semilinear evolution equation:

$$
\left\{\begin{array}{l}
u^{\prime}(t) \in A u(t)+f(t, u(t)), \quad t \in I=[0,1] \\
u(0)=g(u)
\end{array}\right.
$$

By using Theorem 3.3, the problem (4.1) has at least one mild solution $u \in C\left([0,1] ; L^{2}(\Omega)\right)$ provided that hypotheses (3)-(5) and (3.1) hold. 


\section{Competing interests}

The authors declare that they have no competing interests.

\section{Authors' contributions}

All authors read and approved the final manuscript.

\section{Author details}

${ }^{1}$ Department of Mathematics and Physics, Nanjing Institute of Technology, Nanjing, 211100, P.R. China. ${ }^{2}$ Department of Mathematics, Yangzhou University, Yangzhou, 225002, P.R. China.

\section{Acknowledgements}

The authors sincerely thank the reviewers for their valuable suggestions and useful comments that have led to the present improved version of the original manuscript. The research was supported by the Scientific Research Foundation of Nanjing Institute of Technology (No: QKJA2011009).

\section{Received: 12 April 2013 Accepted: 10 June 2013 Published: 1 July 2013}

\section{References}

1. Byszewski, L: Theorems about the existence and uniqueness of solutions of a semilinear evolution nonlocal Cauchy problem. J. Math. Anal. Appl. 162, 494-505 (1991)

2. Byszewski, L: Existence and uniqueness of solutions of semilinear evolution nonlocal Cauchy problem. Zesz. Nauk. Politech. Rzesz., Mat. Fiz. 18, 109-112 (1993)

3. Byszewski, L, Lakshmikantham, V: Theorems about the existence and uniqueness of a solutions of nonlocal Cauchy problem in a Banach space. Appl. Anal. 40, 11-19 (1990)

4. Ntouyas, S, Tsamotas, P: Global existence for semilinear evolution equations with nonlocal conditions. J. Math. Anal Appl. 210, 679-687 (1997)

5. Ntouyas, S, Tsamotas, P: Global existence for semilinear integrodifferential equations with delay and nonlocal conditions. Anal. Appl. 64, 99-105 (1997)

6. Zhu, T, Song, C, Li, G: Existence of mild solutions for abstract semilinear evolution equations in Banach spaces. Nonlinear Anal. 75, 177-181 (2012)

7. Zhu, T, Song, C, Li, G: Existence of mild solutions for nonlocal integro-differential equations in Banach spaces. Adv Differ. Equ. Control Process. 10(1), 57-67 (2012)

8. Aizicovici, S, Gao, Y: Functional differential equations with nonlocal initial condition. J. Appl. Math. Stoch. Anal. 10, 145-156 (1997)

9. Aizicovici, S, Mckibben, M: Existence results for a class of abstract nonlocal Cauchy problems. Nonlinear Anal. 39, 649-668 (2000)

10. Aizicovici, S, Lee, H: Nonlinear nonlocal Cauchy problems in Banach spaces. Appl. Math. Lett. 18, $401-407$ (2005)

11. Xie, C, Zhong, W, Fan, Z: Existence results for nonlinear nonlocal problems in Banach spaces. Appl. Math. Lett. 22, 998-1002 (2009)

12. Xue, X: Nonlinear differential equations with nonlocal conditions in Banach spaces. Nonlinear Anal. 63, 575-586 (2005)

13. Xue, X, Song, G: Perturbed nonlinear evolution inclusions in Banach spaces. Acta. Math. Sci. 15, 189-195 (1995)

14. Xue, X: Nonlocal nonlinear differential equations with a measure of noncompactness in Banach spaces. Nonlinear Anal. 70, 2593-2601 (2009)

15. Zhu, L, Li, G: On a nonlocal problem for semilinear differential equations with upper semicontinuous nonlinearities in general Banach spaces. J. Math. Anal. Appl. 341, 660-675 (2008)

16. Cardinali, T, Rubbioni, P: On the existence of mild solutions of semilinear evolution differential inclusions. J. Math. Anal. Appl. 308, 620-635 (2005)

17. Gori, C, Obukhovskii, V, Ragni, M, Rubbioni, P: Existence and continuous dependence results for semilinear functional differential inclusions with infinite delay. Nonlinear Anal. 51, 765-782 (2002)

18. Benchohra, M, Ntouyas, SK: Existence of mild solutions of second order initial value problems for delay integrodifferential inclusions with nonlocal conditions. Math. Bohem. 127, 613-622 (2002)

19. Benchohra, M, Henderson, J, Ntouyas, SK: Existence results for impulsive multivalued semilinear neutral functional differential inclusions in Banach spaces. J. Math. Anal. Appl. 263, 763-780 (2001)

20. Fu, X, Cao, Y: Existence for neutral impulsive differential inclusions with nonlocal conditions. Nonlinear Anal. 68 3707-3718 (2008)

21. Pazy, A: Semigroups of Linear Operators and Applications to Partial Differential Equations. Springer, New York (1983)

22. Banas, J, Goebel, K: Measure of Noncompactness in Banach Spaces. Lecture Notes in Pure and Applied Math., vol. 60. Dekker, New York (1980)

23. Mönch, $\mathrm{H}$ : Boundary value problems for nonlinear ordinary differential equations of second order in Banach spaces. Nonlinear Anal. TMA 4, 985-999 (1980)

24. Xue, X: Semilinear nonlocal differential equations with measure of noncompactness in Banach spaces. J. Nanjing Univ., Math. Biq. 24, 264-276 (2007)

25. Bothe, D: Multivalued perturbation of $m$-accretive differential inclusions. Isr. J. Math. 108, 109-138 (1998)

26. Kamenskii, M, Obukhovskii, V, Zecca, P: Condensing Multivalued Maps and Semilinear Differential Inclusions in Banach Spaces. De Gruyter Ser. Nolinear Anal. Appl., vol. 7. de Gruyter, Berlin (2001)

27. Zeidler, E: Nonlinear Functional Analysis and Its Applications I: Fixed Point Theorems. Springer, Berlin (1992)

28. Agarwal, R, Meehan, M, O'Regan, D: Fixed Point Theory and Applications, Cambridge Tracts in Math. Cambridge University Press, Cambridge (2001)

29. Granas, A, Dugundji, J: Fixed Point Theory. Springer, New York (2003)

30. Deimling, K: Nonlinear Functional Analysis. Springer, Berlin (1985)

31. Lasota, A, Opial, Z: An application of the Kakutani-Ky-Fan theorem in the theory of ordinary differential equations. Bull. Acad. Pol. Sci., Sér. Sci. Math. Astron. Phys. 13, 781-786 (1965) 
32. Hu, S, Papageorgiou, NS: Handbook of Multivalued Analysis: Theory, vol. I. Kluwer Academic, Dordrecht (1997)

33. Martin, RH: Nonlinear Operators and Differential Equations in Banach Spaces. Wiley, New York (1976)

doi:10.1186/1687-2770-2013-153

Cite this article as: Zhu et al.: Existence results for abstract semilinear evolution differential inclusions with nonlocal conditions. Boundary Value Problems 2013 2013:153.

Submit your manuscript to a SpringerOpen ${ }^{\odot}$ journal and benefit from:

- Convenient online submission

Rigorous peer review

- Immediate publication on acceptance

- Open access: articles freely available online

- High visibility within the field

- Retaining the copyright to your article

Submit your next manuscript at $\boldsymbol{s p r i n g e r o p e n . c o m ~}$ 